\title{
VESICLE RECYCLING AT RIBBON SYNAPSES IN THE FINELY BRANCHED AXON TERMINALS OF MOUSE RETINAL BIPOLAR NEURONS
}

\author{
L. LoGIUDICE, ${ }^{a, c}$ P. STERLING ${ }^{b}$ AND G. MATTHEWS ${ }^{a *}$ \\ ${ }^{a}$ Department of Neurobiology and Behavior, State University of New \\ York, Stony Brook, NY 11794-5230, USA \\ ${ }^{b}$ Department of Neuroscience, University of Pennsylvania, Philadel- \\ phia, PA 19104, USA \\ 'Program in Neuroscience, State University of New York, Stony Brook, \\ NY 11794-5230, USA
}

\begin{abstract}
In retinal bipolar neurons, synaptic ribbons mark the presence of exocytotic active zones in the synaptic terminal. It is unknown, however, where compensatory vesicle retrieval is localized in this cell type and by what mechanism(s) excess membrane is recaptured. To determine whether endocytosis is localized or diffuse in mouse bipolar neurons, we imaged FM4-64 to track vesicles in cells whose synaptic ribbons were tagged with a fluorescent peptide. In synaptic terminals, vesicle retrieval occurred at discrete sites that were spatially consistent over multiple stimuli, indicative of endocytotic "hot spots." Retrieval sites were spatially correlated with fluorescently labeled synaptic ribbons. Electron microscopy (EM) analysis of bipolar cell terminals after photoconversion of internalized FM dye revealed that almost all of the dye was contained within vesicles $\sim 30 \mathrm{~nm}$ in diameter. Clathrin-coated vesicles were observed budding from the plasma membrane and within the cytosol, and application of dynasore, a dynamin inhibitor, arrested membrane retrieval just after the budding stage. We conclude that synaptic vesicles in the fine branches of mouse bipolar axon terminals are retrieved locally near active zones, at least in part via a clathrin-mediated pathway. (c) 2009 IBRO. Published by Elsevier Ltd. All rights reserved.
\end{abstract}

Key words: retina, synaptic transmission, endocytosis, neurotransmitter release, synaptic vesicles.

To conserve the readily releasable pool of vesicles in a presynaptic terminal, vesicle membrane inserted during exocytosis must be retrieved by compensatory endocytosis and then recycled to active zones as newly generated synaptic vesicles (Schweizer and Ryan, 2006; Dittman and Ryan, in press). Ribbon-type synapses in retina and inner ear, which are specialized for rapid and sustained exocytosis, might require specialized mechanisms of membrane retrieval to keep pace. Indeed, images from electron microscopy (EM) indicated that membrane recovery at ribbon synapses of goldfish bipolar neurons and frog saccular hair

*Corresponding author. Tel: +1-631-632-8648; fax: +1-631-632-4858 E-mail address: Gary.G.Matthews@sunysb.edu (G. Matthews).

Abbreviations: DMSO, dimethyl sulfoxide; EGTA, ethylene glycol tetraacetic acid; EM, electron microscopy; HEPES, 4-(2-hydroxyethyl)-1piperazineethanesulfonic acid; NMDG, N-methyl-D-glucamine; PAF, paraformaldehyde; RBP, RIBEYE-binding peptide. cells involves bulk endocytosis, where large endosomes pinch off from the plasma membrane and then produce synaptic vesicles (Lenzi et al., 2002; Paillart et al., 2003; Holt et al., 2003; LoGiudice and Matthews, 2007). This would be consistent with specialization of the endocytosis phase to match the high exocytotic load-quite different from the standard pathway of clathrin-mediated retrieval of single fused vesicles at conventional, non-ribbon synapses. The fish bipolar and frog hair cell showed little evidence for the canonical clathrin-mediated mechanism even though components of this pathway, including clathrin and dynamin, are expressed in some ribbon-containing neurons (Ullrich and Südhof, 1994; von Kriegstein et al., 1999; Sherry and Heidelberger, 2005).

But the goldfish bipolar neurons and frog hair cells were originally chosen for study because of their large size and huge reserve pools of synaptic vesicles $(\sim 900,000$ in the goldfish terminal; von Gersdorff et al., 1996). So we wondered if these large reserves might allow bulk uptake to combine with leisurely replenishment of the releasable pool, whereas smaller terminals with smaller reserve pools might rely on the standard clathrin-mediated mechanism. To answer this, we characterized compensatory endocytosis at a mammalian ribbon-containing neuron, the mouse retinal bipolar cell, whose terminal volume and reserve pool is more than 100 -fold smaller than that of the goldfish. The small size led us to adopt a fluorescence imaging approach for physiological characterization of the vesicle cycle at individual ribbon-type active zones, using a fluorescent peptide to label ribbons and uptake of FM dyes to mark internalized vesicles. Then, we examined the same cell by EM after photoconversion of internalized dye to determine the ultrastructural basis of compensatory endocytosis.

In this way, we addressed several questions about the endocytotic limb of the vesicle cycle at mammalian ribbontype synapses. First, is excess membrane retrieved at discrete locations within the terminal? If so, are these sites stable over multiple rounds of stimulation? Second, where is membrane recaptured with respect to ribbon-type active zones? Third, is membrane initially recovered in bulk or directly as single vesicles? Fourth, do new vesicles immediately enter the releasable pool? Fifth, does clathrin-mediated retrieval play a role in the vesicle cycle? If so, at what stage in the endocytotic pathway is it involved? The answers to these questions suggest that the size of the reserve pool at a ribbon synapse dictates whether compensatory endocytosis occurs in bulk or leads directly to production of new synaptic vesicles via a clathrin-mediated pathway. 


\section{EXPERIMENTAL PROCEDURES}

\section{Bipolar cell isolation}

We used adult male mice of strain C57BL/6J (Taconic, Germantown, NY, USA). Animal use was approved by the Institutional Animal Care and Use Committee of SUNY Stony Brook and was in accord with the United States Public Health Service Policy on Humane Care and Use of Laboratory Animals. The number of animals and their suffering were minimized. A mouse was killed by $\mathrm{CO}_{2}$ inhalation, and both eyes were removed and hemisected. Neural retinas were detached and cut into six pieces. To isolate bipolar cells, retinal pieces were dissociated by mechanical trituration after papain digestion for $\sim 30 \mathrm{~min}$ at room temperature (Heidelberger and Matthews, 1992). Cells were then plated on flame-polished glass coverslips in a solution containing $135 \mathrm{mM}$ $\mathrm{NaCl}, 5 \mathrm{mM} \mathrm{KCl}, 1 \mathrm{mM} \mathrm{MgCl}_{2}, 2.5 \mathrm{mM} \mathrm{CaCl}_{2}$, and $10 \mathrm{mM} \mathrm{HEPES}$ and briefly washed with the same solution prior to recording. All experiments were done at room temperature.

\section{Electrophysiology}

Isolated bipolar cells were identified based on their distinctive morphology (Ghosh et al., 2004). Cells were voltage clamped using an EPC-9 amplifier controlled by Pulse software (HEKA, Bellmore, NY, USA). Bipolar cell bodies were patch clamped, held at $-60 \mathrm{mV}$, and dialyzed with a Cs-gluconate solution (120 mM Cs-gluconate, $10 \mathrm{mM}$ TEA-Cl, $20 \mathrm{mM}$ HEPES, $0.2 \mathrm{mM}$ NMDGEGTA, $3 \mathrm{mM} \mathrm{MgCl}_{2}$, $2 \mathrm{mM} \mathrm{Na} \mathrm{m}_{2}$ ATP, $0.5 \mathrm{mM}$ GTP) containing $35 \mu \mathrm{M}$ fluorescent peptide (fluor-EQTVPVDLSVARPR-COOH; AnaSpec, San Jose, CA, USA) with affinity for the CtBP2 domain of the synaptic ribbon protein RIBEYE (RIBEYE binding peptide, RBP) (Zenisek et al., 2004). The extracellular bath solution contained $135 \mathrm{mM} \mathrm{NaCl}, 5 \mathrm{mM} \mathrm{KCl}, 1 \mathrm{mM} \mathrm{MgCl}_{2}, 2.5 \mathrm{mM} \mathrm{CaCl}_{2}$, and $10 \mathrm{mM}$ HEPES. The $\mathrm{pH}$ of the solution was adjusted to $\sim 7.4$ with $\mathrm{NaOH}$.

\section{Confocal imaging}

All fluorescence images were acquired using an Olympus FV-300 laser-scanning confocal microscope and Fluoview software (Olympus, America, Center Valley, PA, USA). The fluoresceintagged peptide and FM4-64 labeling were sequentially scanned to reduce channel crosstalk. Coordination with the patch-clamp amplifier and programming of sequences of image acquisitions were accomplished using Tiempo software extension to Fluoview (Olympus America, Center Valley, PA, USA). Subsequent analysis and processing of images was carried out in Fluoview, ImageJ (http://rsbweb.nih.gov/ij/), or Igor Pro (WaveMetrics, Lake Oswego, OR, USA) software.

\section{Visualizing retrieval sites}

FM4-64 (20 $\mu \mathrm{M}$; Invitrogen, Carlsbad, CA, USA) was focally applied to cells exposed to $80 \mathrm{mM}$ high $\mathrm{K}^{+}$. To label a small fraction of vesicles, FM4-64 was focally applied to voltage-clamped cells, and a $250 \mathrm{~ms}$ depolarizing stimulus was given after achieving asymptotic intensity of FM4-64 fluorescence in the plasma membrane. Approximately $12 \mathrm{~s}$ after the onset of stimulation, Advasep-7 (Biotium, Hayward, CA, USA) was puffed onto the cell to quickly remove plasma membrane fluorescence, leaving behind trapped dye only. To detect sites of retrieval over multiple rounds of stimulation, FM4-64 internalized on a previous round was photobleached by increasing the $543 \mathrm{~nm}$ laser intensity to $100 \%$ for $\sim 5 \mathrm{~s}$. Dynasore, when used, was diluted to $80-90 \mu \mathrm{M}$ in $0.16 \%$ DMSO. Cells were exposed to dynasore for $\sim 5 \mathrm{~min}$ prior to stimulating cells with high $\mathrm{K}^{+}$in the presence of $\mathrm{FM}$ dye. Control cells were exposed to $0.16 \%$ DMSO.
Fixation, photoconversion, and electron microscopy

For EM, cells were plated on Aclar instead of glass coverslips and were stimulated in the presence of FM1-43 (5 $\mu \mathrm{M}$; Invitrogen) or AM1-43 (5 $\mu \mathrm{M}$; Biotium). Cells were washed and lightly fixed in $4 \%$ paraformaldehyde (PAF) and $0.2 \%$ glutaraldehyde in $0.13 \mathrm{M}$ PB (phosphate buffer)at room temperature for $30 \mathrm{~min}$, then bathed in diaminobenzidine (DAB) for $10 \mathrm{~min}$. Photoconversion was achieved by illuminating with high intensity blue light through a $50 \mathrm{x}$ objective for $20 \mathrm{~min}$ in the presence of diaminobenzidine. Cells were washed and fixed with $2.5 \% \mathrm{PAF}$ and $2.5 \%$ glutaraldehyde in $0.13 \mathrm{M} \mathrm{PB}$ for $30 \mathrm{~min}$ at room temperature, then processed for EM as previously described (Paillart et al., 2003). Uranyl Acetate and Lead Citrate, typically used to stain the sections, were omitted to preserve the contrast between labeled and unlabeled vesicles, unless otherwise noted (Brumback et al., 2004).

\section{Immunolabeling isolated neurons}

Cells were isolated as usual and plated on glass coverslips. They were fixed with $4 \%$ PAF for $15 \mathrm{~min}$ at room temperature then bathed in $0.3 \%$ Triton X-100 for $30 \mathrm{~min}$ at room temperature. Cells were subsequently incubated in primary antibody for $1.0 \mathrm{~h} 15 \mathrm{~min}$ and then in secondary antibody for $1.0 \mathrm{~h}$ at room temperature. Primary antibody: Mouse monoclonal against dynamin-1 (1:250; BD Biosciences, Franklin Lakes, NJ, USA). Secondary antibody: Goat anti-mouse conjugated to AlexaFluor 546 (1:200; Jackson ImmunoResearch, West Grove, PA, USA).

\section{Data presentation}

Data in the text are presented as mean \pm 1 standard error of the mean, and statistical significance in pairwise comparisons was assessed using two-tailed $t$-tests with unequal variance.

\section{RESULTS}

\section{"Hot spots" of membrane retrieval}

To detect sites of membrane retrieval, we depolarized single bipolar cells by focally applying the styryl dye FM4-64 in high $\mathrm{K}^{+}$for several seconds. Approximately $12 \mathrm{~s}$ after the onset of stimulation, the cells were washed with a dye-free external solution containing Advasep-7 to eliminate plasma membrane fluorescence. Trapped FM4-64 was confined to the synaptic terminals and the adjacent axon (Fig. 1A), and was located discretely, suggesting that endocytosis occurred at distinct sites along the plasma membrane (Fig. 1B). To determine whether recovered membrane redistributes throughout the cytosol or remains concentrated near the initial retrieval sites, we imaged the same terminals 5 min after application of Advasep-7 (Fig. 1B, right panel). The fluorescent spots were spatially stable over this interval, indicating that populations of recovered structures remained relatively close to their respective retrieval sites (Fig. 1C).

Are sites of endocytotic activity consistent over multiple stimuli? To address this question, we depolarized individual cells with focal superfusion of high $\mathrm{K}^{+}$plus FM4-64 and then washed with Advasep-7 to reveal the location of trapped fluorescence (Fig. 2A, top panel). Then, we bleached the FM4-64 fluorescence by exposing the cells to high intensity $543 \mathrm{~nm}$ laser light. The cells were then stimulated a second time with high $\mathrm{K}^{+}$plus FM4-64, and after $12 \mathrm{~s}$, they were washed with Advasep-7 (Fig. 2A, bottom panel). The spatial fluorescence patterns produced by each stimulus were highly 

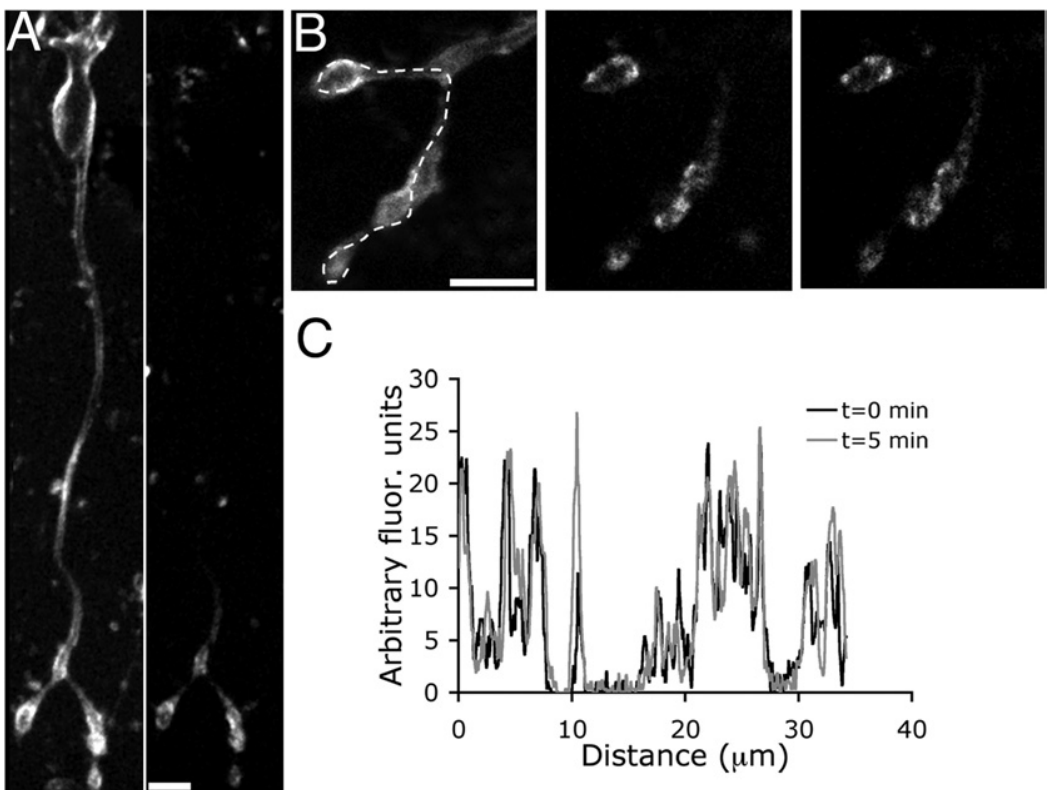

C

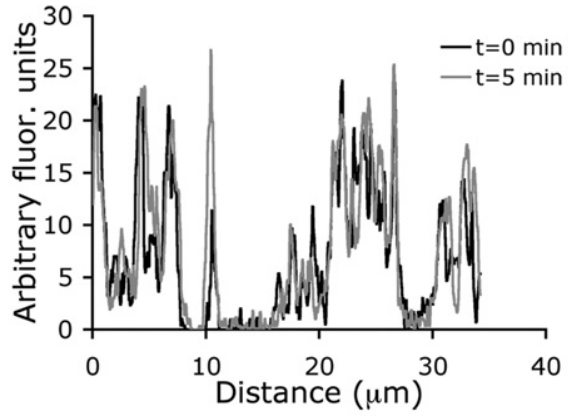

Fig. 1. Activity-dependent membrane retrieval occurs at discrete locations within the terminal. The fluorescence profile remains stable over time. (A) Confocal z-stack of a mouse bipolar cell that was stimulated with high $\mathrm{K}^{+}$plus FM4-64 (left panel). Cell was then washed with Advasep-7 to rapidly remove plasma membrane dye and expose trapped fluorescence (right panel). (B) Zoomed-in images of the same terminals shown in $A$, before washing with Advasep-7 (left panel), immediately following Advasep-7 wash (middle panel), and 5 min later (right panel). (C) Spatial profile under the dashed line in B of the fluorescence immediately following Advasep-7 (black trace) and 5 min later (gray trace).

correlated, indicating that sites of activity-dependent endocytosis are consistent over at least two rounds of stimulation (Fig. 2B, C). Although the stimuli were separated by minutes, and the calcium currents remained comparable in amplitude, the increase in fluorescence after the second stimulus was on average $\sim 30 \%$ less than after the first.

\section{Membrane retrieval occurs near synaptic ribbons}

In Figs. 1, 2, we showed that excess membrane inserted during exocytosis is retrieved at distinct sites along the membrane. Are these retrieval sites spatially related to "hot spots" of vesicle fusion? In retinal bipolar neurons, many of the exocytotic active zones are marked by the presence of synaptic ribbons (LoGiudice et al., 2008; Zenisek, 2008; Midorikawa et al., 2007). Therefore, by marking synaptic ribbons with a fluorescent peptide, and labeling recycling membrane with FM4-64, we could visualize the vesicle cycle with respect to individual active zones in the living cell (LoGiudice et al., 2008). To directly assess whether trapped dye concentrates near ribbons, cells were bathed in high $\mathrm{K}^{+}$and FM4-64 for $90 \mathrm{~s}$ and then washed with Advasep-7 to remove excess membrane staining. Subsequently, cells were dialyzed, via whole-cell patch pipette, with a fluorescent peptide that binds to the ribbon protein RIBEYE (RBP). Sequential confocal scanning revealed that trapped FM dye concentrates near synaptic ribbons within minutes of membrane recovery (Fig. 3A).

To resolve the position of retrieved membrane within $\sim 15 \mathrm{~s}$ of stimulus onset, cells were patch-clamped and dialyzed with the fluorescent peptide prior to introducing FM4-64 into the bath (Fig. 3B). The dye was then focally applied to a voltage-clamped neuron, and after achieving asymptotic intensity of FM4-64 in the membrane, the cell was presented with a $250 \mathrm{~ms}$ depolarizing pulse. FM4-64 was removed from the bath $\sim 12 \mathrm{~s}$ after stimulation, and Advasep-7 was puffed onto the cell to remove residual external dye, leaving the fluorescence trapped in endosomes that were internalized during the post-stimulation interval (Fig. 3B, C). Similar to prolonged stimulation with high $\mathrm{K}^{+}$, a single, short depolarization produced an increase in FM4-64 fluorescence at discrete locations within the synaptic terminals. The resulting spatial fluorescence profile of synaptic ribbons and FM4-64 fluorescence reveals that sites of membrane retrieval are highly correlated with the location of exocytotic active zones in the axon and axon terminals (Fig. 3D).

To compare the percent of the total fluorescence that is retrieved near and away from ribbons, the average FM4-64 fluorescence per pixel was measured within $750 \mathrm{~nm}$ of the plasma membrane in all regions of the terminal cluster, including ribbon and non-ribbon locations. This provided a normalization factor for the overall brightness of a particular cell. Then, the fluorescence per pixel was measured in a square $0.75 \times 0.75 \mu \mathrm{m}$ region of interest centered on a synaptic ribbon, defined by punctate RBP staining, and compared to the fluorescence per pixel at non-ribbon locations. The total area away from ribbons was approximately twice that of the area containing synaptic ribbons. The data graphed in Fig. 3E show that across 10 cells containing 113 synaptic ribbons, the fluorescence per pixel at ribbons was $218 \pm 18 \%$ of the average fluorescence per pixel for the entire terminal, but at non-ribbon locations the fluorescence per pixel averaged $46 \pm 6 \%$ of the total fluorescence per pixel $(P<0.001)$. The result indicates that the primary sites of com- 

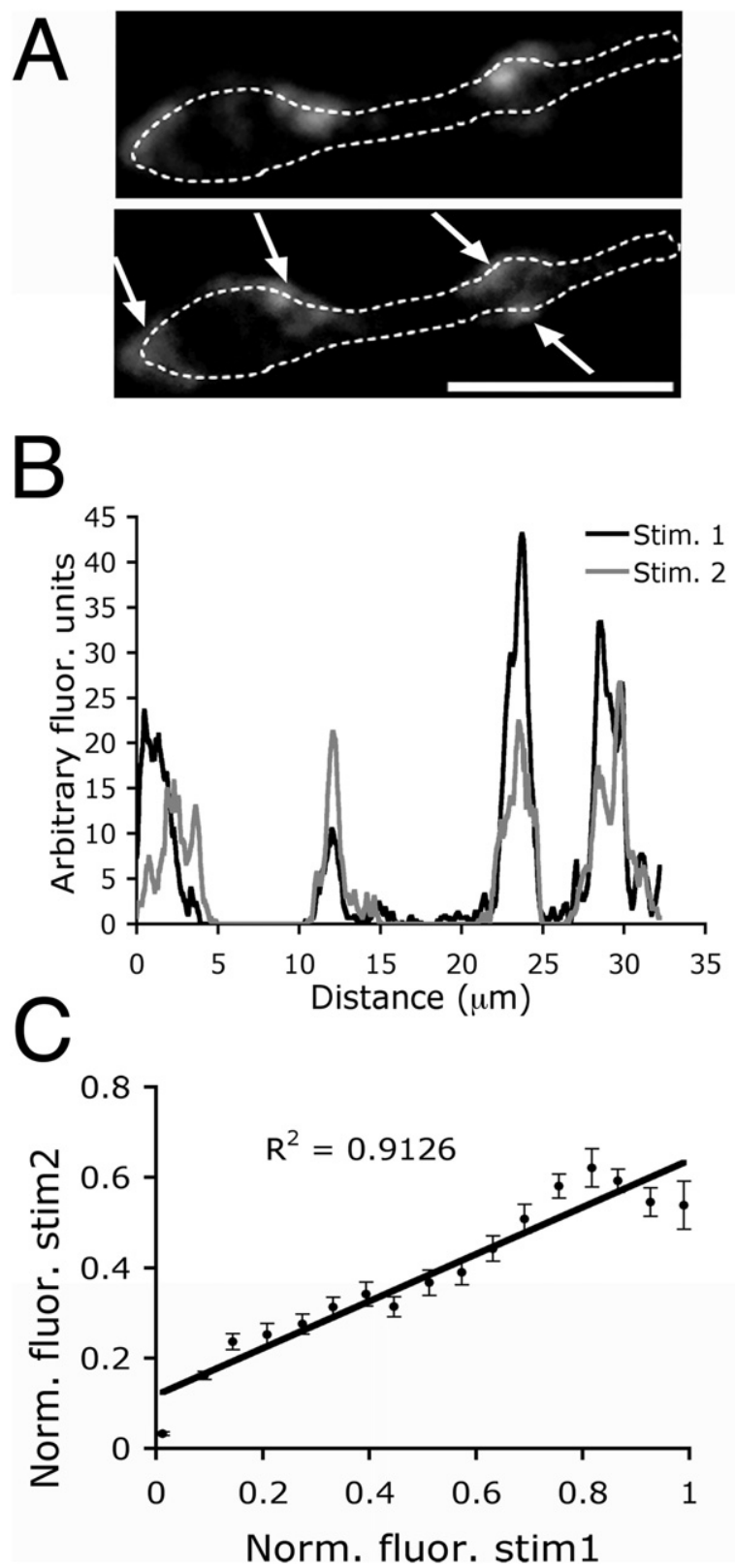

Fig. 2. Membrane is retrieved at "hot spots" within the terminal. (A) Terminals were briefly exposed to FM4-64 and high $\mathrm{K}^{+}$and then washed with Advasep-7 to reveal trapped dye (top panel). The dye was then bleached with $543 \mathrm{~nm}$ laser light. The cell was exposed to a second round of FM4-64 and high $\mathrm{K}^{+}$then washed with Advasep-7 (bottom panel). Scale bar represents $5.0 \mu \mathrm{m}$. (B) Fluorescence intensity profile along the dashed line shown in A for the first (black) and second (gray) stimulus. Endocytosis occurs in similar positions after each stimulus. (C) Graph illustrating the relationship between the fluorescence profiles after the first and second rounds of stimulation (total of 2042 points, $n=4$ cells).

pensatory endocytosis are located near ribbon-type active zones.

\section{Evidence for direct retrieval of synaptic vesicles}

Following brief or sustained stimulation, compensatory endocytosis in goldfish bipolar cell terminals relies heavily on bulk membrane retrieval (Paillart et al., 2003; Holt et al., 2003). In mouse bipolar cells however, single vesicles, not large endosomes, are most frequently labeled with FM1-43 after prolonged stimulation (LoGiudice et al., 2008). To clarify further whether membrane is retrieved in large bites or as single small vesicles, we depolarized cells that were exposed to FM1-43, fixed, photoconverted, and prepared them for EM. In strongly stimulated terminals that were repetitively depolarized with local superfusions of high $\mathrm{K}^{+}$ over the course of $5 \mathrm{~min}$, the majority of photoconverted product was contained within single vesicles with a mean diameter of $\sim 33 \mathrm{~nm}$ (Fig. 4A, F), which is the same as the average diameter of synaptic vesicles in mouse bipolar cell terminals (LoGiudice et al., 2008). In total, $\sim 20 \%$ of vesicles were labeled after $5 \mathrm{~min}$ of repetitive stimulation with high $\mathrm{K}^{+}$, including a subset that recycled back to synaptic ribbons (LoGiudice et al., 2008). To examine retrieval after brief stimulation, cells were patch-clamped and fixed following a single $250 \mathrm{~ms}$ depolarizing voltage-clamp pulse. After brief depolarization, photoconverted product also localized to structures with a size distribution equivalent to that of synaptic vesicles (Fig. 4E, F). A single $250 \mathrm{~ms}$ stimulus resulted in the labeling of $\sim 5 \%$ of the total vesicle population, and even with such brief stimulation, a small number of labeled vesicles appeared to be associated with synaptic ribbons (Fig. 4D), suggesting immediate entry of at least some recently recycled vesicles into the releasable pool.

The immediate appearance of labeled synaptic vesicles in the releasable pool argues for direct production of vesicles during endocytosis, rather than bulk membrane retrieval of large endosomes that later give rise to synaptic vesicles, as was described in goldfish bipolar neurons (Paillart et al., 2003; LoGiudice and Matthews, 2007). To examine this in more detail, we depolarized cells with superfusion of high $\mathrm{K}^{+}$and then fixed during the stimulus to capture the early phases of membrane retrieval. By EM, the plasma membrane of these neurons was crenellated, with numerous omega-shaped invaginations that are similar in size to synaptic vesicles (Fig. 4B, C). Large invaginations were not observed budding from the plasma membrane and labeled cytosolic structures were equivalent in size to synaptic vesicles. Both the invaginations at the plasma membrane and the internalized endosomes were confined to single thin sections in a series, indicating that they are unlikely to be part of larger tubular endosomes. The results suggest that under both strong and weak stimulus conditions, the principal mechanism of endocytosis in mouse bipolar neurons involves the retrieval of small vesicles similar in size to synaptic vesicles, rather than large endosomes.

\section{Evidence for clathrin-mediated retrieval}

A candidate mechanism for such direct retrieval of small vesicles is clathrin-mediated endocytosis, which involves the formation of coated pits and coated vesicles that can be recognized in EM images. However, in the experiments using photoconverted FM dye to label vesicles, presented above, we omitted staining of sections with Uranyl Acetate 

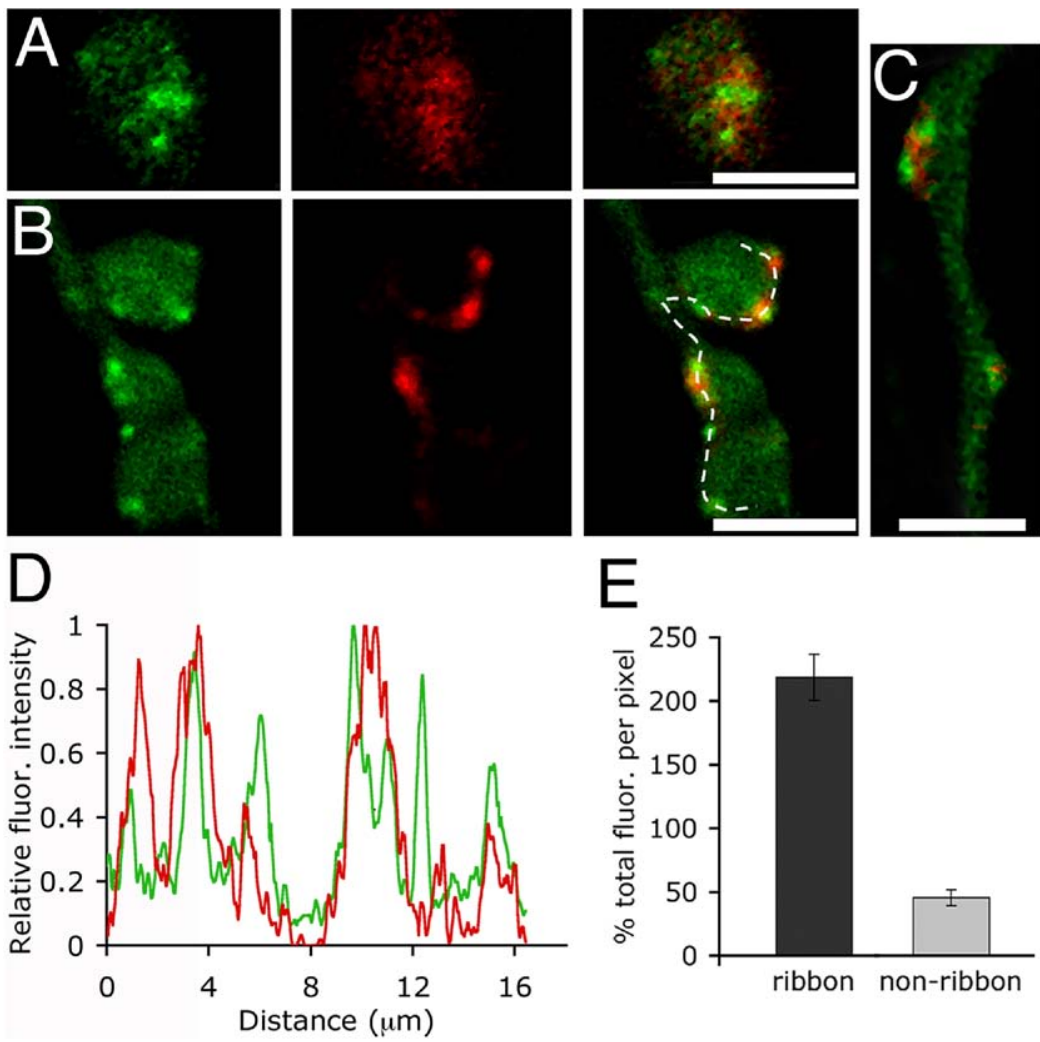

Fig. 3. Sites of endocytosis correlate with the position of synaptic ribbons in the axon and terminals. (A) Confocal images of a live bipolar cell loaded with FM4-64 (red) and RIBEYE-binding peptide (green). To load recycling vesicles, cells were bathed in a high $\mathrm{K}^{+}$solution containing $20 \mu \mathrm{M}$ FM4-64 for $90 \mathrm{~s}$ and washed with Advasep-7. The last panel is an overlay of the red and green channels. Scale bar represents $2.5 \mu \mathrm{m}$. (B) Confocal images of a different terminal loaded with RIBEYE-binding peptide (RBP) (green) via a patch pipette and depolarized from a holding potential of -60 to 0 mV for $250 \mathrm{~ms}$ in the presence of FM4-64. Dye was removed after $\sim 12 \mathrm{~s}$, and the cell was washed with Advasep-7 to expose the location of trapped dye (middle panel). Far right panel is the overlay. Scale bar represents $5.0 \mu \mathrm{m}$. (C) FM4-64 labeling (red) also concentrates around axonal ribbons (green). Scale bar represents $2.5 \mu \mathrm{m}$. (D) Fluorescence intensity profile of synaptic ribbons (green) and FM4-64 (red) along the dashed line shown in B. (E) Graph showing the percent total FM4-64 fluorescence per pixel at ribbon and non-ribbon locations within the axon and terminals $(P<0.001, n=10$ cells). See text for a description of the measurement procedure.

and Lead Citrate in order to preserve the contrast between labeled and unlabeled vesicles. As a result, the low contrast of the unstained sections made it difficult to detect clathrin coats on nascent endocytic profiles in stimulated terminals. Therefore, to better distinguish clathrin cages, we fixed cells in the absence of FM dye and changed our EM protocol to include Uranyl Acetate and Lead Citrate staining of the EM sections. In this material, coated pits and coated vesicles with the classical appearance of a clathrin lattice were commonplace in stimulated terminals and were frequently detected near synaptic ribbons (Fig. 5). From this, we conclude that clathrin is likely to be involved in the initial stages of retrieval in mouse bipolar neurons, similar to synaptic vesicle recycling at conventional synapses.

The GTPase dynamin is thought to play a crucial role in the pinching off of clathrin-coated pits from the plasma membrane to form internalized coated vesicles (Koenig and Ikeda, 1989; Takei et al., 1998; Sweitzer and Hinshaw, 1998; Henley et al., 1999). To determine if dynamin is enriched in synaptic terminals of mouse bipolar cells, we immunolabeled isolated cells using an antibody against dynamin-1. We found intense dynamin immunostaining specifically in the synaptic terminals (Fig. 6A), which is consistent with our EM evidence suggesting an important role for clathrin-mediated endocytosis in membrane retrieval after bouts of exocytosis. To obtain functional evidence that the high level of dynamin at the mouse bipolar cell synapse is important for synaptic vesicle endocytosis, we used the dynamin inhibitor dynasore (Macia et al., 2006), which has previously been shown to strongly block endocytosis at synapses of cultured hippocampal neurons (Newton et al., 2006). In bipolar cell terminals that were exposed to $80 \mu \mathrm{M}$ dynasore prior to and during stimulation, FM4-64 fluorescence remained associated with the plasma membrane of the terminal (Fig. 6B), whereas control cells from the same preparation showed the normal pattern of dye internalization (Fig. 6C). This is consistent with inhibition by dynasore of the scission stage of endocytosis. Curiously, however, the plasma membrane of dynasoretreated terminals continued to fluoresce 8-10 min after removing the dye from the bath (Fig. 6B). By contrast, unstimulated control terminals treated similarly lost plasma fluorescence within seconds after removing external FM dye, as expected from the rapid exit of the dye from the external membrane leaflet. The fact that dynasore-treated 

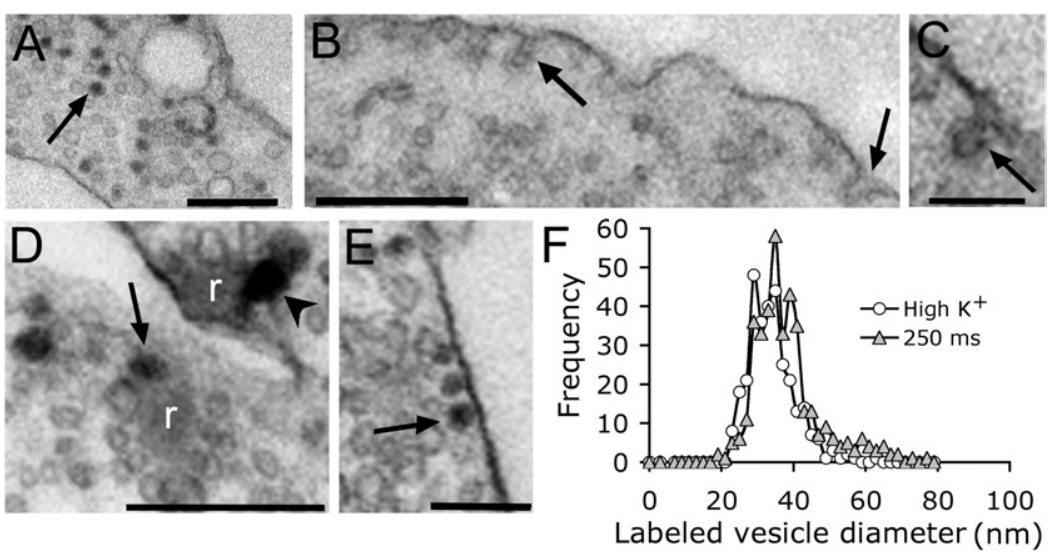

Fig. 4. EM analysis of photoconverted FM1-43 reveals that endosomes the size of single synaptic vesicles are retrieved in response to brief and prolonged stimulation. (A) EM image of a mouse bipolar cell synaptic terminal loaded with FM1-43 in the presence of high $\mathrm{K}^{+}$. Cells were fixed and then photoconverted. Sections were not stained with Uranyl Acetate or Lead Citrate in order to preserve the native contrast between photoconverted and unlabeled vesicles. Dark spots indicate vesicles filled with FM1-43 (e.g., arrow). Scale bar represents $0.5 \mu \mathrm{m}$. (B, C) Cell was fixed before removing high $\mathrm{K}^{+}$from the bath. (B) The plasma membrane appears crenellated with omega-shaped invaginations (arrows). Scale bar represents $0.25 \mu \mathrm{m}$. (C) High magnification image of a budding vesicle. Scale bar represents $0.1 \mu \mathrm{m}$. (D) EM image of two synaptic ribbons ( $r$ ) in a cell that was patch clamped and then stimulated with a single $250 \mathrm{~ms}$ depolarization. The cell was fixed shortly thereafter and photoconverted. Arrow points to a synaptic vesicle that appears to have recycled back to the ribbon. Scale bar represents $0.25 \mu \mathrm{m}$. A larger structure appears adjacent to the other ribbon (arrow head). (E) Three labeled vesicles docked at the membrane (arrow). Scale bar represents $0.125 \mu \mathrm{m}$. (F) Size distribution of labeled vesicles in cells stimulated with high $\mathrm{K}^{+}$(triangles) and a 250 ms depolarizing stimulus (circles). Mean diameter is $\sim 33 \mathrm{~nm}$. Vesicle size was measured in NIH Image.

terminals did not similarly destain indicates that the FM dye was trapped in an internalized compartment, yet remained close to the plasma membrane.

To identify the basis of the trapped, membrane-associated fluorescence, cells that were stimulated in the presence of AM1-43 and dynasore were fixed and photoconverted approximately $3 \mathrm{~min}$ after stimulation, and then prepared for EM. Ultrastructural inspection uncovered a likely explanation for the unusual dye retention near the plasma membrane. Much of the AM1-43 photoproduct was contained within membrane-bound structures that appeared to be arrested in the budding phase of retrieval (Fig. 6D) and sometimes remained connected to the plasma membrane via a thin neck (examples are shown in
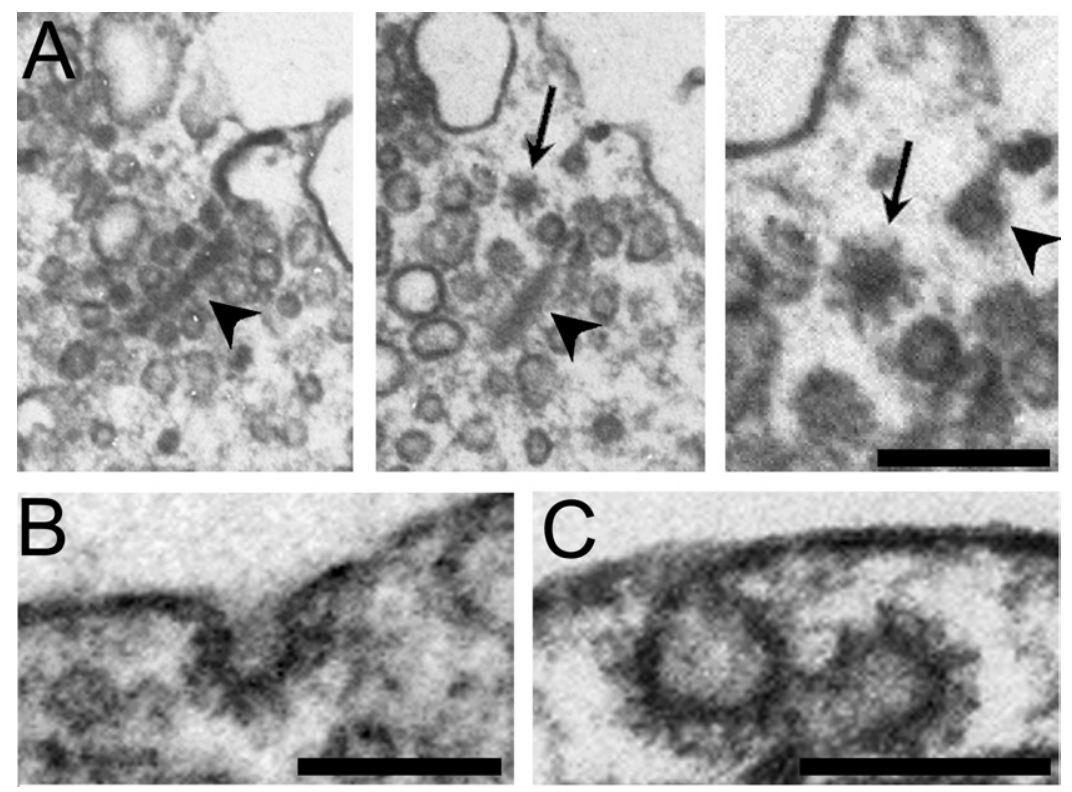

Fig. 5. Clathrin-coated vesicles in mouse bipolar neurons. (A) Serial EM sections of a cell that was patch-clamped and held at $-60 \mathrm{mV}$, then fixed $\sim 30 \mathrm{~s}$ after the patch pipette was removed. The left panel shows a synaptic ribbon (arrow head). The middle panel is the next section in the series and shows a cytoplasmic clathrin-coated vesicle (arrow) adjacent to the synaptic ribbon (arrow head). The panel on the right is a higher-magnification view of the coated vesicle in the middle panel. Another structure, also visible in the left and middle panels, seems to be budding from the membrane (arrow head). (B) EM image of a clathrin-coated vesicle budding from the plasma membrane. The cell was stimulated with high $\mathrm{K}^{+}$for $\sim 30 \mathrm{~s}$ and fixed before removing high $\mathrm{K}^{+}$from the bath. (C) EM image of multiple clathrin coated vesicles in the process of budding. Same cell as in A. Scale bars in $\mathrm{A}-\mathrm{C}$ represent $0.125 \mu \mathrm{m}$. 

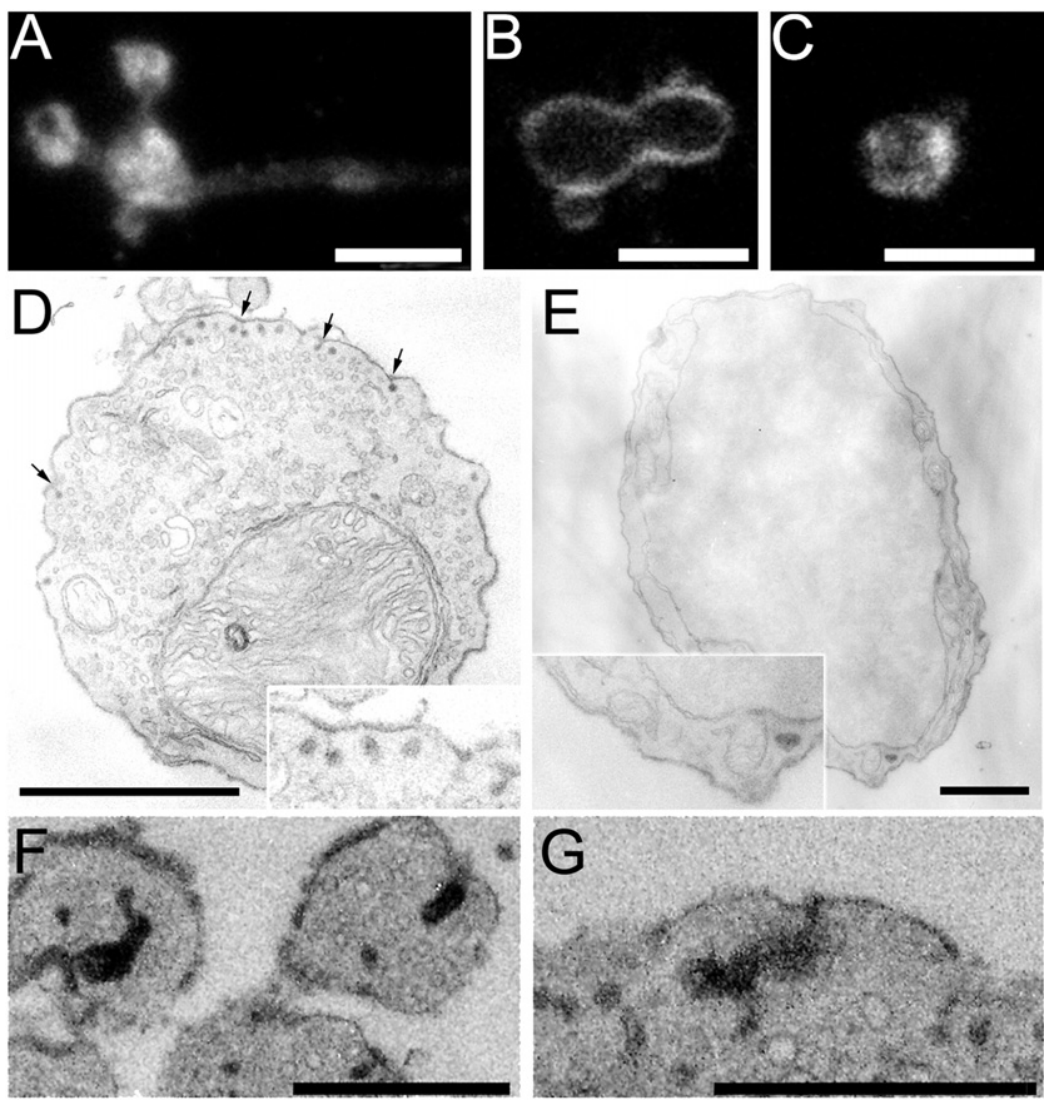

Fig. 6. Membrane retrieval is modified when dynamin GTPase is inhibited by dynasore. (A) Confocal image of an isolated mouse bipolar cell terminal immunolabeled with an antibody against dynamin-1. (B, C) Confocal images of terminals briefly stimulated by high $\mathrm{K}^{+}$in the presence of FM4-64. Control cells were bathed in buffer containing $0.16 \%$ DMSO (C) and experimental cells were bathed in buffer containing $80 \mu \mathrm{M}$ dynasore dissolved in $0.16 \%$ DMSO (B). Images were taken $\sim 5.5 \mathrm{~min}$ after stimulation. Dye appears to be trapped at the membrane in cells exposed to dynasore, while control cells internalized the dye. Scale bars in A-C represent $5.0 \mu \mathrm{m}$. (D) EM image of a cell exposed to $80 \mu \mathrm{M}$ dynasore and stimulated with a $3 \mathrm{~s}$ puff of high $\mathrm{K}^{+}$in the presence of AM1-43. Cell was fixed $\sim 3.0 \mathrm{~min}$ after stimulation and subsequently photoconverted. Small vesicles containing photoconverted AM1-43 appear "stuck" on the membrane (arrows). Scale bar represents $1 \mu \mathrm{m}$. Inset shows a higher-magnification view of some of the labeled structures. (E) The cell body of the same bipolar neurons shown in A lacks structures containing photoconverted AM1-43. Scale bar=1 $\mu \mathrm{m} .(F, G)$ Large structures containing photoconverted AM1-43 appear to be attached to the plasma membrane. Scale bars represent $0.5 \mu \mathrm{m}$.

the inset of Fig. 6D). No similar labeled structures were observed in the cell body of stimulated, dynasore-treated bipolar neurons (Fig. 6E) or in the axon (not shown), which indicates that the membrane-associated structures are specifically related to membrane retrieval at the synapse. In dynasore-treated terminals fixed 3 min after stimulation, there were $0.71 \pm 0.10$ labeled vesicles per $\mu \mathrm{m}$ of terminal perimeter that were attached to the plasma membrane (15 terminals in six thin sections from two cells), but in control cells exposed only to DMSO, the average number of labeled vesicles associated with the membrane was $0.1 \pm 0.04$ vesicles per micrometer of terminal perimeter (seven terminals in five thin sections from two cells; $P<0.0005)$. Therefore, dynasore inhibits the complete internalization of vesicles during compensatory endocytosis at the synapse, but apparently at a stage after the interior of the endocytosed vesicle is no longer connected to the extracellular space. Otherwise, the dye would not have been trapped, and the vesicles would not have been marked with dark photoproduct.

In addition to the synaptic vesicle-like structures attached to the membrane, larger endocytic structures were also observed to be associated with the membrane in dynasore-treated terminals. Fig. 6F, G show EM images of terminals that were stimulated in the presence of AM1-43 and dynasore, and then fixed and photoconverted $3 \mathrm{~min}$ later. Larger, irregular structures appeared near the membrane in dynasore-treated terminals, and these complex structures also contained photoproduct from AM dye that did not destain during the 3 min interval between dye removal and fixation. In stimulated terminals exposed to dynasore, the density of large labeled endosomes $(>100$ $\mathrm{nm}$ in the longest dimension) was $0.59 \pm 0.13 / \mu \mathrm{m}^{2} 2(15$ terminals in six thin sections from two cells), compared with $0.09 \pm 0.09 / \mu \mathrm{m}^{2}$ in DMSO control cells (seven terminals in five thin sections from two cells; $P<0.03$ ). The formation of these large endosomes may also reflect inhibition by dynasore of the normal process of budding and pinching off membrane in bites the size of synaptic vesicles.

The overall density of labeled endosomes of all sizes was not significantly different in dynasore-treated and control terminals (control: $8.4 \pm 2.5 / \mu \mathrm{m}^{2}$; dynasore: $13.4 \pm$ 
$\left.1.9 / \mu \mathrm{m}^{2} ; P=0.14\right)$. Therefore, dynasore does not block endocytosis per se in mouse bipolar cell synapses, but seems to arrest the process of complete separation from the plasma membrane and release of newly formed vesicles.

\section{DISCUSSION}

\section{Direct recycling vs bulk endocytosis}

We have found that mouse bipolar cell terminals retrieve the membrane added during neurotransmitter release by direct recovery of small vesicles. Furthermore, recently recycled vesicles were found to be associated with synaptic ribbons, even after brief stimulation with a single voltage-clamp depolarization, which indicates that at least some newly retrieved vesicles are reused quickly in mouse bipolar cells. This scheme contrasts with goldfish bipolar cells and frog saccular hair cells, where membrane is recovered largely by means of bulk retrieval (Lenzi et al., 2002; Holt et al., 2003; Paillart et al., 2003), possibly operating in parallel with clathrin-dependent endocytosis (Jockusch et al., 2005). The large endosomes resulting from bulk endocytosis in goldfish bipolar terminals appear to arise first after stimulation and then later give rise to synaptic vesicles (LoGiudice and Matthews, 2007). However, the direct route of compensatory endocytosis we observed in mouse bipolar cells more closely resembles endocytosis in synapses containing conventional active zones, such as retinal amacrine cells (Paillart et al., 2003; LoGiudice and Matthews, 2007) and hippocampal neurons (Granseth et al., 2006).

Why are different endocytic mechanisms used in terminals of goldfish vs. mouse bipolar neurons? Perhaps the

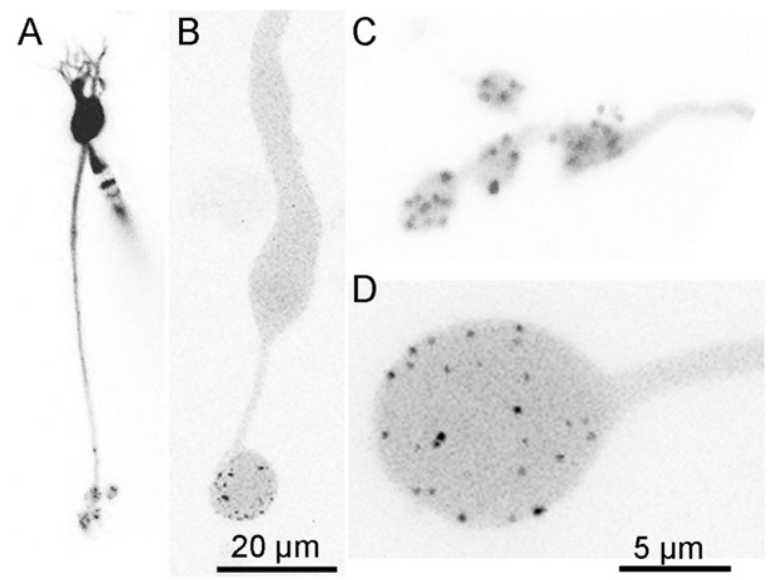

Fig. 7. Comparison of synaptic terminals of living bipolar neurons isolated from mouse and goldfish retina. Cells were filled via a wholecell patch pipette with fluorescein-conjugated RBP and imaged using confocal microscopy. Images are z-axis projections of optical sections through the entire cell. (A) An isolated mouse bipolar neuron. The patch pipette is visible, attached to the cell body. (B) An isolated goldfish bipolar neuron at the same magnification as $A$. The patch pipette was removed in this instance prior to imaging. Scale bar applies to A and B. (C) Mouse bipolar cell terminal boutons at higher magnification. Different cell from A. (D) Goldfish bipolar cell terminal at the same magnification as C. Different cell from B. Scale bar in D applies to $\mathrm{C}$. key is simply the relative volume of the synaptic terminals, and hence the size of the pool of preformed synaptic vesicles held in reserve. For comparison, Fig. 7 shows examples of isolated mouse and goldfish bipolar neurons, filled with fluorescent RBP to reveal cell shape and mark ribbons in the terminals. In agreement with reconstructions of bipolar neurons from EM images of serial sections of mouse retina (Tsukamoto et al., 2001), dissociated mouse bipolar cell somata are $\sim 5 \mu \mathrm{m}$ in diameter, and their synaptic endings consist of a spray of small boutons 1.5$2.5 \mu \mathrm{m}$ in diameter (Fig. 7A, C). In contrast, goldfish bipolar cell bodies are $>10 \mu \mathrm{m}$ in diameter, and their bulbous terminals are 10-14 $\mu \mathrm{m}$ across (Fig. 7B, D). The giant terminals of goldfish bipolar neurons contain up to 900,000 synaptic vesicles (von Gersdorff et al., 1996), which is $>100$-fold more than in single terminals of mouse bipolar cells. Therefore, mouse bipolar cells depend more strongly on immediate resupply of new synaptic vesicles to maintain the pool of releasable vesicles, while goldfish bipolar cells can rely on their large pool of pre-existing vesicles to replenish the releasable pool.

Comparison of the degree of labeling of the total vesicle population after stimulation of the 2 cell types supports this interpretation. We found here that even a single 250 ms depolarization under voltage clamp labels $\sim 5 \%$ of the total vesicle population in mouse bipolar cell terminals, but in goldfish terminals, repetitive stimulation for $>10 \mathrm{~min}$ with dozens of calcium action potentials-each lasting $\sim 200$ ms (Zenisek and Matthews, 1998)—caused labeling of only $\sim 10 \%$ of the total vesicle pool (Paillart et al., 2003). This demonstrates directly that immediate recycling of synaptic vesicles is more significant for the small terminals of mouse bipolar cells than the large terminals of goldfish bipolar cells.

Immediate recycling of synaptic vesicles, without bulk retrieval, also occurs in ribbon synapses of cone photoreceptors from lizard retina (Rea et al., 2004), even though these terminals contain large numbers of vesicles $(\sim 250,000$; Rea et al., 2004), much like goldfish bipolar cell terminals. Why, then, do cones not use the more leisurely approach to vesicle retrieval that appears to be used in goldfish bipolar neurons? In darkness, cones are depolarized and have a high rate of ongoing vesicle release, which is reduced in a graded manner to encode light intensity during illumination (Choi et al., 2005). However, ON-type bipolar cells, like the giant-terminal cells of goldfish, produce a transient burst of release at the onset of depolarization, followed by slower sustained release (von Gersdorff et al., 1998; Singer and Diamond, 2003). Therefore, cones have unusually high demand for vesicle resupply compared to bipolar cells, and their synaptic terminals are specialized in a number of ways to ensure that high release rates can be sustained in darkness. For example, synaptic ribbons in photoreceptors are much larger than in bipolar neurons (Sterling and Matthews, 2005), and they tether many more immediately releasable vesicles. In addition, nearly all of the large stock of vesicles in cone terminals are rapidly mobile and participate in the vesicle cycle (Rea et al., 2004), so cones have only a small pool of vesicles held in reserve. So, we 
suggest that in addition to terminal size, the requirements for continuous release can also dictate the use of an immediate recycling scheme in ribbon synapses.

\section{Clathrin-mediated endocytosis}

Retrieval of single synaptic vesicles is mediated by a clathrin-dependent pathway in conventional synapses (Takei et al., 1996; Shupliakov et al., 1997; Granseth et al., 2006), and in keeping with this mechanism, we frequently observed coated vesicles budding from the plasma membrane in mouse bipolar cell terminals following stimulation. Also, we found that the dynamin inhibitor, dynasore, modified the endocytotic process. Ultrastructural examination of dynasore-treated terminals revealed AM1-43-labeled structures the size of synaptic vesicles that were suspended in the budding phase of endocytosis, indicating a role for dynamin in the recovery of membrane added during synaptic exocytosis. In addition, some labeled vesicles that were fully internalized were also observed in terminals exposed to dynasore, which may indicate that block of dynamin by dynasore was incomplete in our experiments. Alternatively, the fully internalized vesicles in the presence of dynasore may reflect a dynamin-independent form of endocytosis operating in parallel, such as the mechanism described at the rat calyx of Held by Xu et al. (2008).

The incidence of large, pleiomorphic invaginations attached to the membrane also increased significantly in cells exposed to dynasore. What process might give rise to these structures? Synaptic terminals possess multiple mechanisms of endocytosis in addition to clathrin-dependent retrieval of single vesicles (Miller and Heuser, 1984; for reviews see Matthews, 2004; Rizzoli and Betz, 2005; Cousin, 2009; Dittman and Ryan, in press). One possibility is that the large invaginations in the presence of dynasore reflect a separate form of endocytic retrieval that is revealed when clathrin-dependent endocytosis is inhibited. In conventional synaptic terminals of cultured cerebellar granule cells, activity-dependent bulk retrieval is triggered rapidly in response to strong stimuli (Clayton et al., 2008), which cause sufficient exocytosis to overwhelm the limited capacity of the clathrin-mediated pathway. Furthermore, this form of bulk endocytosis also depends on dynamin GTPase activity, because it is inhibited by dynasore (Clayton et al., 2009), which induces formation of tubular structures similar to those we observed. In our experiments, the large structures remained attached to the membrane in the presence of dynasore, which suggests that full retrieval of the bulk endosomes is also dynamin-dependent in bipolar cell terminals. This is also consistent with the build-up of tubular invaginations of the plasma membrane at synapses of dynamin-1-knock out mice (Ferguson et al., 2007; Hayashi et al., 2008). These intriguing similarities suggest that the pleiomorphic invaginations in the presence of dynasore arise from the same process of activity-dependent bulk endocytosis characterized by Clayton et al. (2008, 2009) in conventional synapses. An alternative explanation, however, is that excess membrane is simply unable to pinch off when clathrin-mediated endocytosis is inhibited and accumulates in some instances beneath the surface, resulting in the formation of large invaginations without requiring a separate clathrin-independent pathway.

\section{Mechanisms regulating the balance between bulk and clathrin-mediated endocytosis}

How might the balance be tilted toward clathrin-mediated endocytosis at small ribbon terminals, but toward bulk endocytosis at large ribbon synapses? The answer is not yet clear, and a wide variety of factors could regulate endocytic pathways (see Dittman and Ryan, in press, for a recent review). Nevertheless, an important player in the choice of endocytosis mechanisms at a given synapse under particular stimulation conditions may be the GTPase dynamin, which is involved in both clathrin-mediated retrieval of single vesicles (Koenig and Ikeda, 1989; Takei et al., 1998; Sweitzer and Hinshaw, 1998; Henley et al., 1999) and activity-dependent bulk endocytosis (Clayton et al., 2009). Several molecular interactions are known to influence dynamin's participation in endocytosis, including calcineurin-mediated dephosphorylation, which is required for the dynamin-syndapin interaction implicated in bulk retrieval (Clayton et al., 2009). Therefore, dynamin might serve as a dephosphorylation-dependent trigger for alternative retrieval mechanisms in response to increased presynaptic calcium, which serves as an index of exocytotic load.

In addition, clathrin-mediated compensatory endocytosis at synapses may be saturable at moderate exocytotic loads, perhaps because of limited availability of molecular components of the clathrin pathway, including dynamin. This notion has led to the suggestion (e.g., Rizzoli and Betz, 2005) that bulk endocytosis may be an "emergency response" observed only with stimulation that exceeds the ability of clathrin-mediated endocytosis to keep up. However, at the giant ribbon synapses of goldfish bipolar cells, bulk endocytosis is observed after brief stimulation with single calcium action potentials (Paillart et al., 2003; also see re-analysis presented in LoGiudice and Matthews, 2007), and it is the fast component of endocytosis that remains after blockade of clathrin-dependent endocytosis (Jockusch et al., 2005). This is consistent with bulk retrieval being a normal, rapid component of compensatory endocytosis at this synapse. We also point at that, if anything, the exocytotic load is greater as a proportion of plasma membrane surface area in the smaller terminals of mouse bipolar cells, which have $\sim 8$ ribbons per terminal (LoGiudice et al., 2008) and a terminal surface area of $10-20 \mu \mathrm{m}^{2}$. By comparison, bulbous terminals of goldfish bipolar cells have $\sim 60$ ribbons (von Gersdorff et al., 1996) but a surface area of $300-500 \mu \mathrm{m}^{2}$. Because endocytosis seems to be local (see below), each ribbon can be considered to a first approximation to behave as an exocytotic/ endocytotic unit, and thus there is no reason to suppose that the load imposed on compensatory endocytosis is greater in the large terminals of goldfish bipolar cells. We suggest, then, that the use of direct vs. indirect recycling mechanisms at morphologically distinct ribbon synapses is likely to depend on differences in molecular components 
and/or signaling mechanisms, rather than simply the load imposed by the amount of exocytosis.

\section{Endocytosis occurs at hot spots near ribbons}

In our experiments, we have also addressed several questions about the location of compensatory endocytosis in mouse bipolar cells. First, compensatory membrane retrieval, visualized with FM4-64, occurs at discrete locations within axons and synaptic terminals. By optically tracking FM dye over time, we showed that recaptured membrane remains concentrated near retrieval sites over the course of several minutes. Moreover, sites of membrane retrieval were consistent over multiple rounds of endocytosis, indicative of endocytotic "hot spots" in mouse bipolar cell synaptic terminals.

Second, the hot spots of compensatory retrieval are spatially correlated with the position of synaptic ribbons, a structural hallmark of exocytotic active zones in bipolar neurons (LoGiudice et al., 2008; Zenisek, 2008). Previously, EM images in photoreceptor cells and hair cells supported the idea that excess membrane inserted during fusion is locally recycled near synaptic ribbons. In cone and rod photoreceptors, retrieved vesicles appeared to form on the membrane lateral to synaptic ribbons (Gray and Pease, 1971; Sterling and Matthews, 2005), and in frog hair cells, elongated omega profiles and clathrincoated pits were observed adjacent to synaptic bodies (Lenzi et al., 1999). However, the sites of membrane retrieval had not been directly visualized in living ribbon synapses. By concurrently visualizing fluorescently labeled synaptic ribbons and FM4-64 uptake in living neurons, we have shown that sites of endocytosis are indeed related to synaptic ribbon locations and hence, active zones. This is analogous to the layout of retrieval sites in the neuromuscular junction, where sites of vesicle fusion and endocytosis are closely associated in space (Teng et al., 1999; Teng and Wilkinson, 2000). Thus, local recycling at active zones is a shared feature of conventional and ribbon-type synapses. As at conventional synapses, no ultrastructural specializations are apparent at ribbons that would restrict endocytosis to the regions near the ribbon, so the local nature of retrieval at ribbons may simply reflect the high efficiency of endocytosis in recovering vesicle components from the plasma membrane near the site of fusion.

Acknowledgments-Supported by NIH grants EY003821 (G.M.) and EY008124 (P.S.). We thank Jian Li and Wendy Akmentin for electron microscopy.

\section{REFERENCES}

Brumback AC, Lieber JL, Angleson JK, Betz WJ (2004) Using FM1-43 to study neuropeptide granule dynamics and exocytosis. Methods 33:287-294.

Choi SY, Borghuis BG, Rea R, Levitan ES, Sterling P, Kramer RH (2005) Encoding light intensity by the cone photoreceptor synapse. Neuron 48:555-562.

Clayton EL, Anggono V, Smillie KJ, Chau N, Robinson PJ, Cousin MA (2009) The phospho-dependent dynamin-syndapin interaction triggers activity-dependent bulk endocytosis of synaptic vesicles. J Neurosci 29:7706-7717.
Clayton EL, Evans GJ, Cousin MA (2008) Bulk synaptic vesicle endocytosis is rapidly triggered during strong stimulation. J Neurosci 28:6627-6632.

Cousin MA (2009) Activity-dependent bulk synaptic vesicle endocytosis-a fast, high capacity membrane retrieval mechanism. Mol Neurobiol 39:185-189.

Dittman J, Ryan TA (in press) Molecular circuitry of endocytosis at nerve terminals. Annu Rev Cell Dev Biol, in press.

Ferguson SM, Brasnjo G, Hayashi M, Wölfel M, Collesi C, Giovedi S, Raimondi A, Gong LW, Ariel P, Paradise S, O'Toole E, Flavell R, Cremona O, Miesenböck G, Ryan TA, De Camilli P (2007) A selective activity-dependent requirement for dynamin 1 in synaptic vesicle endocytosis. Science 316:570-574.

Ghosh KK, Bujan S, Haverkamp S, Feigenspan A, Wässle H (2004) Types of bipolar cells in the mouse retina. J Comp Neurol 469: $70-82$.

Granseth B, Odermatt B, Royle SJ, Lagnado L (2006) Clathrin-mediated endocytosis is the dominant mechanism of vesicle retrieval at hippocampal synapses. Neuron 51:773-786.

Gray EG, Pease HL (1971) On understanding the organisation of the retinal receptor synapses. Brain Res 35:1-15.

Hayashi M, Raimondi A, O'Toole E, Paradise S, Collesi C, Cremona O, Ferguson SM, De Camilli P (2008) Cell- and stimulus-dependent heterogeneity of synaptic vesicle endocytic recycling mechanisms revealed by studies of dynamin 1-null neurons. Proc Natl Acad Sci U S A 105:2175-2180.

Heidelberger R, Matthews G (1992) Calcium influx and calcium current in single synaptic terminals of goldfish retinal bipolar neurons. J Physiol 447:235-256.

Henley JR, Cao H, McNiven MA (1999) Participation of dynamin in the biogenesis of cytoplasmic vesicles. FASEB J 13:S243-S247.

Holt M, Cooke A, Wu MM, Lagnado L (2003) Bulk membrane retrieval in the synaptic terminal of retinal bipolar cells. J Neurosci 23: 1329-1339.

Jockusch WJ, Praefcke GJ, McMahon HT, Lagnado L (2005) Clathrindependent and clathrin-independent retrieval of synaptic vesicles in retinal bipolar cells. Neuron 46:869-878.

Koenig JH, Ikeda K (1989) Disappearance and reformation of synaptic vesicle membrane upon transmitter release observed under reversible blockage of membrane retrieval. J Neurosci 9:3844-3860.

Lenzi D, Crum J, Ellisman MH, Roberts WM (2002) Depolarization redistributes synaptic membrane and creates a gradient of vesicles on the synaptic body at a ribbon synapse. Neuron 36:649659.

Lenzi D, Runyeon JW, Crum J, Ellisman MH, Roberts WM (1999) Synaptic vesicle populations in saccular hair cells reconstructed by electron tomography. J Neurosci 19:119-132.

LoGiudice L, Matthews G (2007) Endocytosis at ribbon synapses. Traffic 8:1123-1128.

LoGiudice L, Sterling P, Matthews G (2008) Mobility and turnover of vesicles at the synaptic ribbon. J Neurosci 28:3150-3158.

Macia E, Ehrlich M, Massol R, Boucrot E, Brunner C, Kirchhausen T (2006) Dynasore, a cell-permeable inhibitor of dynamin. Dev Cell 10:839-850.

Matthews $G$ (2004) Cycling the synapse: scenic versus direct routes for vesicles. Neuron 44:223-226.

Midorikawa M, Tsukamoto Y, Berglund K, Ishii M, Tachibana M (2007) Different roles of ribbon-associated and ribbon-free active zones in retinal bipolar cells. Nat Neurosci 10:1268-1276.

Miller TM, Heuser JE (1984) Endocytosis of synaptic vesicle membrane at the frog neuromuscular junction. J Cell Biol 98:685698.

Newton AJ, Kirchhausen T, Murthy VN (2006) Inhibition of dynamin completely blocks compensatory synaptic vesicle endocytosis. Proc Natl Acad Sci U S A 103:17955-17960.

Paillart C, Li J, Matthews G, Sterling P (2003) Endocytosis and vesicle recycling at a ribbon synapse. J Neurosci 23:4092-4099. 
Rea R, Li J, Dharia A, Levitan ES, Sterling P, Kramer RH (2004) Streamlined synaptic vesicle cycle in cone photoreceptor terminals. Neuron 41:755-766.

Rizzoli SO, Betz WJ (2005) Synaptic vesicle pools. Nat Rev Neurosci 6:57-69.

Schweizer FE, Ryan TA (2006) The synaptic vesicle: cycle of exocytosis and endocytosis. Curr Opin Neurobiol 16:298-304.

Sherry DM, Heidelberger R (2005) Distribution of proteins associated with synaptic vesicle endocytosis in the mouse and goldfish retina. J Comp Neurol 484:440-457.

Shupliakov O, Low P, Grabs D, Gad H, Chen H, David C, Takei K, De Camilli P, Brodin L (1997) Synaptic vesicle endocytosis impaired by disruption of dynamin-SH3 domain interactions. Science 276:259-263.

Singer JH, Diamond JS (2003) Sustained Ca2+ entry elicits transient postsynaptic currents at a retinal ribbon synapse. J Neurosci 23:10923-10933.

Sterling P, Matthews G (2005) Structure and function of ribbon synapses. Trends Neurosci 28:20-29.

Sweitzer SM, Hinshaw JE (1998) Dynamin undergoes a GTP-dependent conformational change causing vesiculation. Cell 93:1021-1029.

Takei K, Haucke V, Slepnev V, Farsad K, Salazar M, Chen H, De Camilli P (1998) Generation of coated intermediates of clathrinmediated endocytosis on protein-free liposomes. Cell 94:131-141.

Takei K, Mundigl O, Daniell L, De Camilli P (1996) The synaptic vesicle cycle: a single vesicle budding step involving clathrin and dynamin. J Cell Biol 133:1237-1250.

Teng H, Cole JC, Roberts RL, Wilkinson RS (1999) Endocytic active zones: hot spots for endocytosis in vertebrate neuromuscular terminals. J Neurosci 19:4855-4866.
Teng H, Wilkinson RS (2000) Clathrin-mediated endocytosis near active zones in snake motor boutons. J Neurosci 20:7986-7993.

Tsukamoto Y, Morigiwa K, Ueda M, Sterling P (2001) Microcircuits for night vision in the mouse retina. J Neurosci 21:8616-8623.

Ullrich B, Südhof TC (1994) Distribution of synaptic markers in the retina: implications for synaptic vesicle traffic in ribbon synapses. J Physiol Paris 88:249-257.

von Gersdorff H, Sakaba T, Berglund K, Tachibana M (1998) Submillisecond kinetics of glutamate release from a sensory synapse. Neuron 21:1177-1188.

von Gersdorff H, Vardi E, Matthews G, Sterling P (1996) Evidence that vesicles on the synaptic ribbon of retinal bipolar neurons can be rapidly released. Neuron 16:1221-1227.

von Kriegstein K, Schmitz F, Link E, Südhof TC (1999) Distribution of synaptic vesicle proteins in the mammalian retina identifies obligatory and facultative components of ribbon synapses. Eur J Neurosci 11:1335-1348.

Xu J, McNeil B, Wu W, Nees D, Bai L, Wu LG (2008) GTP-independent rapid and slow endocytosis at a central synapse. Nat Neurosci 11:45-53.

Zenisek D (2008) Vesicle association and exocytosis at ribbon and extraribbon sites in retinal bipolar cell presynaptic terminals. Proc Natl Acad Sci U S A 105:4922-4927.

Zenisek D, Horst NK, Merrifield C, Sterling P, Matthews G (2004) Visualizing synaptic ribbons in the living cell. J Neurosci 24: 9752-9759.

Zenisek D, Matthews G (1998) Calcium action potentials in retinal bipolar neurons. Vis Neurosci 15:69-75. 